\title{
Efeito do processamento por micro-ondas nas propriedades físicas, físico-químicas e reológicas do grão de trigo e sua farinha
}

\author{
Effect of microwave processing on the physical, physicochemical and rheological properties of \\ wheat grain and its flour \\ Efecto del procesamiento por microondas sobre las propiedades físicas, fisicoquímicas y reológicas \\ del grano de trigo y su harina
}

Flávio Martins Montenegro

ORCID: https://orcid.org/0000-0003-0476-4764 Instituto de Tecnologia de Alimentos, Brasil E-mail: flavio@ital.sp.gov.br

Raquel Bragatto Heffernan

ORCID: https://orcid.org/0000-0001-7512-9229 Instituto de Tecnologia de Alimento, Brasil

E-mail: raquelbragatto@gmail.com

Antonio Marsaioli Júnior

ORCID: https://orcid.org/0000-0002-4305-3466 Instituto de Tecnologia de Alimento, Brasil

E-mail: tonymars@ital.sp.gov.br

Michele Nehemy Berteli

ORCID: https://orcid.org/0000-0002-9765-1947 Instituto de Tecnologia de Alimento, Brasil

E-mail:berteli@gmail.com

Pedro H. Campelo

ORCID: https://orcid.org/0000-0002-5137-0162 Universidade Federal do Amazonas, Brasil

E-mail: pedrocampelo@ufam.edu.br

Maria Teresa Pedrosa Silva Clerici

ORCID: https://orcid.org/0000-0002-8445-336X Universidade Estadual de Campinas, Brasil E-mail: mclerici@unicamp.br

\begin{abstract}
Resumo
Um sistema piloto para aplicação de micro-ondas em alimentos, de frequência de 2,45 GHz, e capacidade de geração de micro-ondas com potências que variam de $100 \mathrm{~W}$ a $3000 \mathrm{~W}$ foi utilizado para avaliar os efeitos desta tecnologia nas características físicas, físico-químicas e reológicas dos grãos de trigo, farinha de trigo e seus respectivos glútens. Amostras de trigo ( $600 \mathrm{~g}$ por tratamento) foram condicionadas até a umidade de $23 \%$ e expostas às micro-ondas nas potências de $100 \mathrm{~W}, 450 \mathrm{~W}$ e $750 \mathrm{~W}$ (TM1, TM 2 e TM 3, respectivamente) até que esta atingisse $16 \%$ e tiveram suas características comparadas com o trigo controle (TC), sem tratamento. Os resultados de rendimento de moagem e volume específico dos grãos não apresentaram diferenças significativas $(p \leq 0,05)$. Estes trigos foram moídos, gerando as farinhas FM 1, FM 2, FM 3 e FC (provenientes de TM1, TM 2, TM 3 e TC, respectivamente). Os resultados de amido danificado das farinhas dos trigos tratados por micro-ondas e a FC não apresentaram diferença significativa entre as suas médias. As farinhas FM1, FM 2 e FM 3 mostraram reduções significativas nos valores de glúten úmido e seco, quando comparadas com a FC, porém seus índices de glúten não se mostraram diferentes estatisticamente. Com exceção da temperatura de pasta, as demais propriedades de pasta (viscosidade máxima, Viscosidade mínima, quebra e viscosidade final) apresentaram-se diferentes significativamente, ao nível de $5 \%$ de significância, e um comportamento progressivo do perfil de viscosidade. A distribuição do tamanho médio das partículas acumulada à $90 \%$, para as farinhas FM 1, FM 2 e FM 3 foram maiores que a acumulada da farinha FC e diferentes significativamente, sendo confirmado pelos maiores valores dos seus diâmetros médios $\left(\mathrm{d}_{4,3}\right)$. A polidispersibilidade das partículas (Span), variou de 1,81 a 1,94, e não apresentou diferença significativa entre todas as amostras, porém devido ao valor obtido ter sido superior a 1 , este indica de que as amostras são polidispersas.
\end{abstract}

Palavras-chave: Micro-ondas; Moagem; Trigo em grão; Farinha de trigo; Propriedades de pasta; Propriedades físicas; Glúten. 


\begin{abstract}
A pilot system for the application of microwaves to foods, with a frequency of $2.45 \mathrm{GHz}$, and microwave powers ranging from $100 \mathrm{~W}$ to $3000 \mathrm{~W}$ was used to evaluate the effects of this technology on physical, physicochemical and rheological characteristics of wheat grains, wheat flour and their respective gluten. Wheat samples (600 g per treatment) were conditioned to moisture of $23 \%$ and exposed to microwaves in the powers of $100 \mathrm{~W}, 450 \mathrm{~W}$ and $750 \mathrm{~W}$ (TM 1 , TM 2 and TM 3, respectively) until it reached $16 \%$ and had their characteristics compared with control wheat (TC), without treatment. The results of milling yield and specific volume of grains did not show significant differences $(\mathrm{p} \leq 0.05)$. These wheats were ground, generating flours FM 1, FM 2, FM 3 and FC (from TM1, TM 2, TM 3 and TC, respectively). The results of wheat flour damaged starch from wheat-treated and FC showed no significant difference between their averages. Flours FM1, FM 2 and FM 3 showed significant reductions in the values of wet and dry gluten, when compared to the FC, but their gluten indexes were not statistically different. With the exception of the paste temperature, other pasting properties (maximum viscosity, minimum viscosity, breakdown and final viscosity) were significantly different, at the level of 5\% significance, and presented a progressive behavior of the viscosity profile. The size distribution of the particle, accumulated at 90\%, for flours FM 1, FM 2 and FM 3 were larger than the accumulated flour of FC and different significantly, confirmed by the higher values of their mean diameters (d4,3). The polydispersity of the particles (Span), ranged from 1.81 to 1.94, and there was no significant difference between all samples, however due to the value obtained being greater than 1, this indicates that the samples are polydispersed.
\end{abstract}

Keywords: Microwave; Milling; Wheat; Wheat flour; Pasting properties; Physical properties; Gluten.

\title{
Resumen
}

Un sistema piloto para la aplicación de microondas, de frecuencia 2,45 GHz y capacidad de generación de microondas con potencias entre $100 \mathrm{~W}$ y $300 \mathrm{~W}$, fue utilizado para evaluar los efectos de esta tecnología en las características físicas, fisicoquímicas y reológicas de los granos de trigo, harina de trigo y sus glutens. Las muestras de trigo (600g por tratamiento) fueron acondicionadas a una humedad de $23 \%$ y luego expuestas en las potencias de microondas de 100 W, $450 \mathrm{~W}$ y $750 \mathrm{~W}$ (TM 1, TM 2 y TM 3, respectivamente) hasta llegar a 16\%, siendo comparadas con un control de trigo (TC) sin tratamiento. Los resultados del rendimiento de moliendas y el volumen especifico de los granos no presentaron diferencias significativas $(\mathrm{p} \leq 0,05)$. Luego, estos trigos fueron molidos generando las harinas FM1, FM 2, FM3 y FC (provenientes de TM1, TM2, TM3 y TC, respectivamente). Los resultados sobre el almidón dañado de las harinas de trigo tratadas por microondas y FC no presentaron diferencias significativas en sus promedios. Las harinas FM 1, FM 2, y FM 3 mostraron una reducción significativa en sus valores de gluten húmedo y seco en comparación con FC, sin embargo, sus índices de gluten no se mostraron estadísticamente diferentes. Excepto la temperatura de pasta, las demás propiedades de pasta (viscosidad máxima, viscosidad mínima, quiebra y viscosidad final) presentaron diferencias significativas, a un nivel de significancia de 5\%, y un comportamiento progresivo del perfil de viscosidad. La distribución del tamaño promedio de partículas acumulada al 90\%, para las harinas FM 1, FM 2 y FM 3 fueron mayores a las de la harina FC, y diferentes significativamente, siendo confirmado por los mayores valores de sus diámetros medios (d4,3). La polidispersibilidad de las partículas (Span), varió entre 1.81 y 1.94, no presentando diferencias significativas entre todas las muestras, no obstante, debido a que el valor obtenido fue superior a 1, este indica que las muestras son polidispersas.

Palabras clave: Microondas; Molienda; Grano de trigo; Harina de trigo; Propiedades de pasta; Propiedades físicas; Gluten.

\section{Introdução}

O trigo é o segundo cereal mais produzido no mundo, entretanto quando se analisa o destino ao consumo humano este ocupa a primeira posição, representando aproximadamente $20 \%$ do total de proteínas e calorias ingeridas no mundo (International Grains Council, 2018; Nirmal et al., 2017). Devido a importância de se garantir a segurança alimentar e minimizar perdas, as operações unitárias executadas durante a etapa de pós-colheita possuem grande importância na disponibilidade e viabilidade tecnológica deste cereal (Kumar \& Kalita, 2017; Payne, 2002).

Por ser um produto sujeito às oscilações de condições climáticas na época de sua colheita, um dos aspectos mais importantes de controle nesta etapa é a umidade do trigo no exato momento em que é colhido (Baloch, 2001). Por muitas vezes antecipa-se a colheita para que chuvas intensas não possam afetar a qualidade nutricional do grão colhido e que precisará ser seco, a fim de garantir uma umidade adequada para permanecer armazenado por tempos prolongados até sua utilização. Recomenda-se a faixa de 10 a $13 \%$ de umidade para seu armazenamento (Baloch, 2001; Chang et al., 2011; Hagstrum et al., 2012; Payne, 2002).

O sistema de secagem a ser escolhido deverá considerar a umidade que o grão apresenta no momento de sua colheita, 
visto que para grãos colhidos com alta umidade requerem secagem rápida a fim de se evitar o início de processos germinativos, que acarretará perdas nutricionais e potencial tecnológico importantes, além do desenvolvimento de contaminação microbiológica, levando a formação de micotoxinas, e contaminação por insetos (Baloch, 2001). Somando a isto, dependendo da intensidade da secagem, ou seja, necessidade de altas temperaturas por tempos prolongados, tipo de combustível empregado e processo empregado, os danos causados nesta etapa acabam sendo irreversíveis, como queima do grão, perda de propriedades nutricionais, potencial reológico e tecnológico prejudicados, incorporação de odores indesejáveis e compostos tóxicos aos grãos, que continuarão presentes ao longo do processamento e transformação do trigo em farinha e demais produtos obtidos durante sua moagem (Hagstrum et al., 2012).

Agentes químicos são bastante utilizados para controle e prevenção de infestações por insetos durante a etapa de póscolheita. Dentre os mais utilizados estão inseticidas e os fumigantes, que necessitam manejo com cuidados especiais em sua utilização, além de possuírem limites máximos permitidos, pois podem promover riscos à saúde de quem consome e quem os utiliza. Devido aos riscos presentes no uso dos agentes químicos, cada vez mais busca-se alternativas de ingredientes naturais que promovam os mesmos benefícios e processos alternativos que garantam a sanidade dos grãos por tempos prolongados (Hagstrum et al., 2012; Khamis et al., 2011; Kumar \& Kalita, 2017).

Desta forma métodos físicos, que no passado possuíam o uso industrial restrito para aplicação em alimentos devido aos altos custos operacionais, voltaram a ser alternativas tecnológicas atraentes e limpas, pela isenção de produtos químicos e vêm sendo estudados contra infestações, inativar enzimas indesejáveis, garantir aumento da vida de prateleira e obter benefícios tecnológicos pelo uso destas tecnologias. Dentre os métodos físicos incluem-se radiação por infravermelho, radiações gama, aquecimento por rádio frequência, aquecimento ôhmico e micro-ondas (Datta \& Almeida, 2014; Li et al., 2017; Liu \& Ma, 2016; Patil et al., 2016; Wang \& Lu, 2013; Xiqiang et al., 2014; Zhang et al., 2018).

As micro-ondas são caracterizadas como radiação eletromagnética que acontecem no espectro entre $300 \mathrm{MHz}$ e 300 $\mathrm{GHz}$, comprimentos de onda variam de $1 \mathrm{~m}$ e $1 \mathrm{~mm}$, respectivamente. Entre as frequências alocadas para aquecimento temos as de $915 \mathrm{MHz}$ e de $2450 \mathrm{MHz}$, correspondendo respectivamente a $32,8 \mathrm{~cm}$ e 12,25 cm. A frequência de $5800 \mathrm{MHz}$, com comprimento de onda de 5,2 cm, também é usada em situações específicas, como por exemplo, em laboratórios (Berteli, 2005; Vadivambal et al., 2007).

O aquecimento por micro-ondas obtém-se pela absorção das micro-ondas pelos materiais que possuem a habilidade de converter a radiação em calor. A esta habilidade é dada o nome de potencial dielétrico. Cada material possui propriedades dielétricas específicas que determinam sua maior ou menor capacidade de conversão da radiação em calor. Estas propriedades são dependentes de fatores como conteúdo de umidade presente no material, a frequência de micro-ondas aplicada, conteúdo de sais, dentre outros fatores (Bansal et al., 2015).

Esta tecnologia possui, além da economia de tempo, a maior vantagem que é o fato de não gerar resíduos aos materiais irradiados ou danos ao meio ambiente e por este motivo é considerada uma energia limpa, característica cada vez mais valorizada por consumidores (Bansal et al., 2015; Patil et al., 2016; Vadivambal et al., 2007).

O uso da radiação por micro-ondas vem aumentando consideravelmente, sendo empregado, e com ótimos resultados, na diminuição das infestações por insetos, inativação enzimática, controle germinativo, estabilização de farelo (Chang, Stell \& Clerici, 2011; Vadivambal, Jayas \& White, 2007). Landriscina et al. (2017), em seu trabalho, credita à tecnologia de radiação por micro-ondas, alterações nas proteínas presentes no trigo, tornando seu glúten amigável (gluten-friendly ${ }^{\mathrm{TM}}$ ), ou seja, apresentando características apropriadas para o consumo por celíacos.

Diante do que foi levantado, o presente estudo possui os objetivos de avaliar o efeito da radiação por micro-ondas nas características físicas, físico-químicas, reológicas dos trigos, farinhas e dos glútens obtidos. 


\section{Metodologia}

\subsection{Matéria-prima}

Para a condução do trabalho foi utilizado trigo (Triticum aestivum L.), produzido em Arapongas-PR, Brasil, de coordenadas geográficas $23^{\circ} 25^{\prime} 10^{\prime \prime}$ latitude sul e $51^{\circ} 25^{\prime} 28^{\prime \prime}$ longitude oeste. O material foi caracterizado em seu recebimento e continha 11,28 $\pm 0,05 \mathrm{~g} / 100 \mathrm{~g}$ de umidade, $13,37 \pm 0,13 \mathrm{~g} / 100 \mathrm{~g}$ de proteína, $80 \mathrm{~kg} / \mathrm{hL}$ de peso hectolítrico (PH) e $78 \%$ de seus grãos foram classificados como duros, pelo grau de dureza através da análise de Single-Kernel Characterization System SKCS (AACCI, 2010).

\subsection{Preparo e condicionamento do trigo}

As etapas de preparo da amostra e o condicionamento do grão de trigo seguiram os procedimentos descritos nos métodos AACCI $n^{\circ}$ 26-10.02 e 26-95.01 (AACCI, 2010). A fim de se garantir um bom rendimento de moagem, para a amostra denominada trigo controle (TC), elevou-se a umidade do grão de trigo para $16 \%$, recomendada para trigos duros, previamente a etapa de moagem. Para os trigos em que se aplicou a radiação por micro-ondas (TM), a umidade foi elevada para $23 \%$, simulando uma situação de colheita realizada sob condições chuvosas, com o objetivo de alcançar a umidade de 16 \% após o processamento por micro-ondas. O tempo de têmpera padronizado para as amostras foi de $24 \mathrm{~h}$, onde os trigos condicionados foram hermeticamente fechados em embalagem plástica e mantidos em temperatura ambiente, para serem processados no dia seguinte.

\subsection{Sistema piloto de secagem por micro-ondas}

O processamento do trigo em grão foi realizado em equipamento piloto multifuncional destinado ao estudo da aplicação de micro-ondas em alimentos, com frequência de 2,45 GHz, conforme apresentado na Figura 1. Este sistema é capaz de geração de micro-ondas com potências que variam de $100 \mathrm{~W}$ a $3000 \mathrm{~W}$. Possui, acoplado ao sistema de transmissão de micro-ondas, dois wattímetros (Dual Directional Power Monitor - GA3004-3F), com potência máxima de medição de 3 kW e que permitem as leituras das potências transmitidas e refletidas (Clerici et al., 2019). As temperaturas podem ser lidas durante o processamento através de 4 fibras ópticas inseridas na cavidade e dispostas no porta amostras e que foram cobertas pelo leito do trigo, e que estão ligadas ao aquisitor de temperatura Luxtron - Fot Lab Kit. Adicionalmente ao sistema gerador de micro-ondas, o sistema possui instalada uma célula de fluxo de carga para acompanhamento da perda de massa da amostra durante o processamento, além de um sistema auxiliar de geração de ar quente para arrastar a umidade retirada da amostra irradiada para fora da cavidade, evitando condensação em seu interior. A temperatura do ar de quente de entrada gerado por este sistema foi de $58{ }^{\circ} \mathrm{C}$ e a velocidade do ar de arraste na saída da cavidade se encontrava na faixa de 1,3 a 1,6 m/s. 
Figura 1. Visão lateral e frontal da cavidade aplicadora de micro-ondas, alimentação horizontal do material, sendo: 1) cavidade de tratamento; 2) entrada e saída de material; 3) entrada para micro-ondas; 4) entrada de ar com filtro de micro-ondas; 5) porta amostra; 6) célula de carga; 7) conjunto de fibras ópticas.

Visão frontal

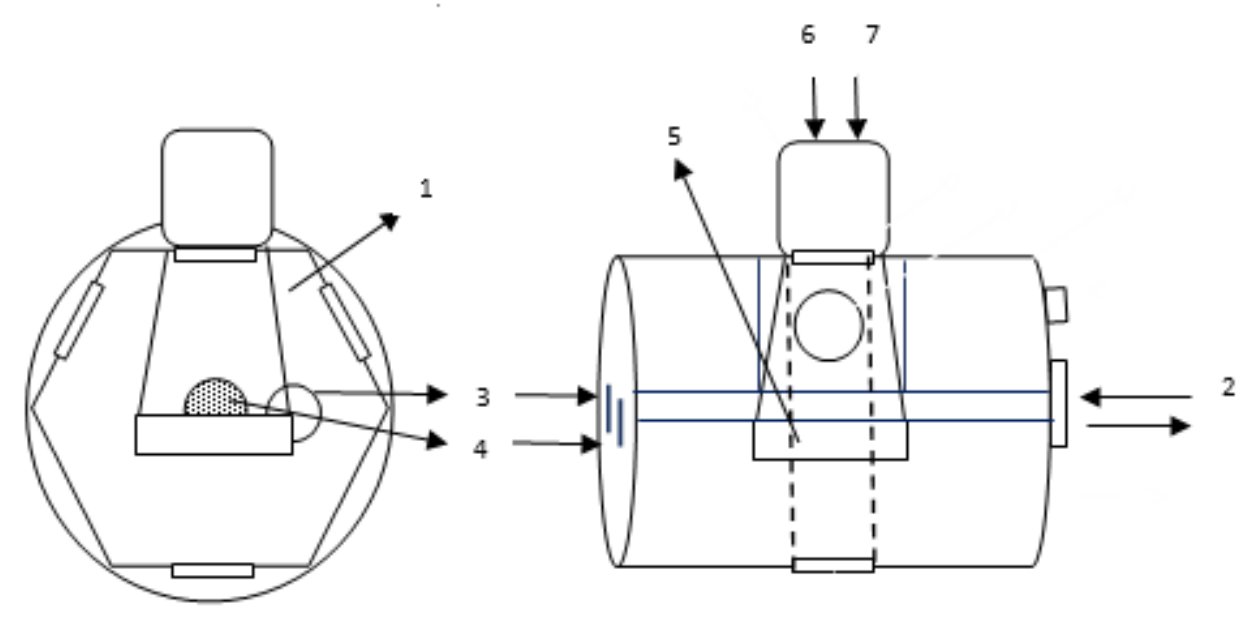

Fonte: Autores.
Visão lateral

7 


\subsection{Avaliação da qualidade tecnológica do trigo e suas farinhas}

Os trigos foram analisados antes e após processamento por micro-ondas quanto ao teor de umidade, segundo método AACCI n 44-15.02 (AACCI, 2010) e volume específico dos grãos, determinado através do volume ocupado por $100 \mathrm{~g}$ de trigo em proveta de $250 \mathrm{~mL}$. Estas análises foram realizadas em triplicata para cada tratamento.

As farinhas de trigo obtidas no trabalho foram avaliadas quanto ao teor de amido danificado (método AACCI 76-33.01). A análise de glúten, quanto aos teores de glúten úmido, glúten seco e glúten index foram determinados de acordo com metodologia AACCI 38-12.02. As propriedades de pasta foram determinadas pela metodologia oficial da AACCI 76-21.01, adotando-se o perfil de temperatura Standard 1 do equipamento. Todas as análises foram realizadas em triplicata para cada tratamento (AACCI, 2010).

A distribuição do tamanho de partículas e o diâmetro médio das partículas realizadas nas farinhas de trigo foram determinadas em analisador de distribuição de tamanho de partículas, modelo LA-950-V2 (Horiba Instruments, Kyoto, Japão) com sistema de difração de raios laser, dispersando as farinhas de trigo estudadas em etanol absoluto 99,5\%. O diâmetro médio das partículas foi determinado segundo o diâmetro de De Brouckere $\left(\mathrm{d}_{4,3}\right)$, que se caracteriza como o diâmetro médio de uma esfera com o mesmo volume da partícula medida. A distribuição do tamanho de partículas é caracterizada pelo diâmetro de partículas acumuladas na distribuição $10 \%, 50 \%$ e $90 \%$ do total das partículas medidas. Também é obtido o índice de polidispersibilidade das partículas (Span), a partir da equação 1, que representa o quanto existe de variação da dispersão de tamanho das partículas em cada amostra.

$$
\text { Span: } \frac{D 90 \%-D 10 \%}{D 50 \%}
$$

onde: D10 \%, D50 \% e D90 \% correspondem aos diâmetros a 10, 50 e 90\% da distribuição cumulativa das amostras.

\subsection{Análise estatística}

Os resultados, obtidos a partir de pelo menos triplicata analítica, tiveram a análise de variância (ANOVA) das suas médias realizada no software SISVAR 5.6 Build 86, sendo que a diferença significativa foi calculada para $p \leq 0,05$ (Tabelas A. 1 até A.16, do Apêndice A). Quando significativo, o teste de agrupamento de médias (Scott-Knott) foi utilizado para se determinar diferenças estatísticas entre si. A escolha pelo teste de Scott-Knott foi porque ele exclui médias pareadas, permitindo determinar a diferenciação entre os tratamentos através da estatística de verossimilhança (Ferreira, 2011).

\section{Resultados e Discussão}

\subsection{Umidade, volume específico e rendimento de moagem do trigo}

A Tabela 2 apresenta os resultados das umidades após condicionamento e após processamento por micro-ondas, bem como os resultados dos volumes específicos dos trigos tratados e os resultados obtidos no rendimento de moagem. Os resultados de umidade após condicionamento e após tratamento por micro-ondas foram inferiores aos estabelecidos para os processos alvo, conforme apresentados na Tabela 1. Haviam sido determinadas, através de cálculos de condicionamento, as umidades de $23 \%$ para os trigos alcançarem após condicionamento e $16 \%$ para a umidade final após os tratamentos por micro-ondas. As umidades obtidas após condicionamento variaram de 21,32 a 22,04 \%, enquanto após processamento as umidades das amostras variaram de 15,00 a $15,43 \%$. O TC apresentou umidade de $15,33 \%$. Estes resultados não foram considerados impeditivos para a condução da sequência do trabalho e realização das análises de qualidade dos materiais.

Os volumes específicos dos grãos de trigo sem tratamento e tratados por micro-ondas, nas diferentes potências variaram de 1,37 a $1,39 \mathrm{~mL} / \mathrm{g}$ e não apresentaram diferenças significativas pela análise de variância (ANOVA) entre os tratamentos $(\mathrm{p} \leq$ 
0,05), indicando que mesmo com o aumento da potência aplicada não ocorreu expansão física dos grãos submetidos aos tratamentos.

Os resultados de moagem obtidos pelas amostras foram similares e a análise de variância (ANOVA), para $p \leq 0,05$, não apresentaram diferença significativas para os diferentes tratamentos, indicando que para as potências utilizadas (100 a $750 \mathrm{~W}$ ) não influenciaram os rendimentos de farinha obtidas.

O rendimento de moagem nos tratamentos realizados variou de 69,49 a 69,83 \% e não houve diferença significativa entre o TC e os trigos TM 1, TM 2 e TM 3, que sofreram a ação das micro-ondas. Estes resultados vão de encontro com os resultados obtidos por Vadivambal et al. (2007), que aplicou micro-ondas em trigo com a finalidade de avaliar o efeito na desinfestação do mesmo. No referido estudo foram utilizadas potências situadas entre 400 e $500 \mathrm{~W}$ em amostras de trigo com diferentes teores proteicos (13,8 a 14,1\%) e teor de umidade que variou de 14 a $16 \%$. Os valores de rendimento de moagem, variaram de 76,5 a 77,4 \%, porém não apresentaram diferenças significativas entre os diferentes tratamentos. A diferença entre os entre os rendimentos de moagem, dos dois estudos, pode ter ocorrido em decorrência de amostras serem de origens diferenciadas e pelo equipamento, também diferente ao que foi utilizado no presente trabalho.

Tabela 2 - Caracterização dos grãos de trigo quanto as suas umidades e volumes específicos e rendimento de moagem após condicionamento e após processamento por micro-ondas.

\begin{tabular}{|c|c|c|c|c|}
\hline \multirow{2}{*}{ Análises } & \multicolumn{4}{|c|}{ Amostras $^{1}$} \\
\hline & TC & TM 1 & TM 2 & TM 3 \\
\hline \multicolumn{5}{|c|}{ Umidade dos trigos em grão } \\
\hline $\begin{array}{l}\text { Umidade após condicionamento (g/100 } \\
\mathrm{g})\end{array}$ & $15,33 \pm 0,08$ & $21,40 \pm 0,04$ & $22,04 \pm 0,12$ & $21,32 \pm 0,14$ \\
\hline $\begin{array}{l}\text { Umidade após processamento por } \\
\text { micro-ondas }(\mathrm{g} / 100 \mathrm{~g})\end{array}$ & na & $15,43 \pm 0,13$ & $15,14 \pm 0,12$ & $15,00 \pm 0,11$ \\
\hline \multicolumn{5}{|c|}{ Volume específico do trigo em grão } \\
\hline $\begin{array}{l}\text { Volume Específico } \\
\qquad(\mathrm{mL} / \mathrm{g})\end{array}$ & $1,37 \pm 0,01^{\mathrm{ns}}$ & $1,37 \pm 0,01^{\mathrm{ns}}$ & $1,39 \pm 0,01^{\mathrm{ns}}$ & $1,37 \pm 0,01^{\mathrm{ns}}$ \\
\hline \multicolumn{5}{|c|}{ Moagem } \\
\hline $\begin{array}{c}\text { Rendimento (耳) } \\
(\%)\end{array}$ & $69,83 \pm 0,18^{\text {ns }}$ & $69,80 \pm 0,16^{\text {ns }}$ & $69,72 \pm 0,15^{\mathrm{ns}}$ & $69,49 \pm 0,61^{\mathrm{ns}}$ \\
\hline
\end{tabular}

${ }^{1} \mathrm{TC}$ - trigo controle; TM 1: trigo tratado com potência de $100 \mathrm{~W}$; TM 2: trigo tratado com potência de $450 \mathrm{~W}$; TM 3: trigo tratado com potência de $750 \mathrm{~W}$. Resultados apresentados como média \pm desvio padrão; na: não aplicável; ns: diferença entre médias na mesma linha não foi significativa pela análise de variância (ANOVA) $(\mathrm{p} \leq 0,05)$. Fonte: Autores.

\subsection{Amido danificado, análise de glúten, propriedades de pasta e distribuição do tamanho de partícula das farinhas de} trigo

A Tabela 3 apresenta os resultados das análises de amido danificado, análise de glúten, propriedades de pasta e da distribuição do tamanho médio de partícula das farinhas de trigo obtidas a partir dos trigos TC, TM1, TM 2 e TM 3. Os resultados de amido danificado presente nas farinhas originadas dos trigos tratados foram comparados com o trigo controle e ficaram situados na faixa de 5,54 a 6,24 \%. Através da análise de variância (ANOVA), constatou-se que não houve diferença significativa entre os resultados para $p \leq 0,05$. Os valores obtidos indicam que o processo por micro-ondas, apesar dos materiais terem sido expostos a tempos e potências aplicadas diferentes, não ocasionou alterações significativas de amido danificado durante o 
processo de moagem ao qual foram submetidas as amostras. Em geral, para moagens industriais, trigos duros geram farinhas com teores de amido danificado situados entre 6 e $12 \%$, sem que esta porcentagem afete a qualidade tecnológica das farinhas produzidas. Resultado para farinha refinada também estão em concordância com os obtidos por Bressiani (2019), que obteve uma farinha refinada com 5,95\% de amido danificado (Cauvain, 2015).

Os resultados para a análise do glúten indicam que a radiação por micro-ondas provocou alterações significativas $(\mathrm{p} \leq 0,05)$, através da análise de agrupamento de médias (Scott-Knott), para as farinhas FM 2 e FM 3 (450 W/18 min e 750W/10 min, respectivamente). A alteração no teor de glúten úmido (GU) e glúten seco (GS) foi maior para a farinha obtida de trigo tratado com a maior potência (FM 3), onde este apresentou 23,6 \% de glúten úmido e 8,0 \% de glúten seco. A farinha FM 1 (100 W/54 min) não apresentou alteração significativa para o teor de glúten úmido e índice de glúten (IG) em relação a FC, sem tratamento por micro-ondas, e obteve o maior teor de glúten seco, quando comparado com todos os tratamentos, incluindo a FC. O índice de glúten não apresentou diferença significativa para todos os tratamentos, indicando que o glúten presente na farinha de trigo, mesmo sendo submetidos a gradativas radiações por micro-ondas, continuou com alta qualidade e valores de IG superiores à 96,8.

De um modo geral, a análise de glúten indica que a medida em que ocorre aumento de potência aplicada nos tratamentos dos trigos, ocorre uma desnaturação proteica que provoca alterações significativas, ao nível de $5 \%$ de significância, retratada nos teores de glúten úmido e glúten seco obtidos pelas farinhas obtidas a partir destes trigos. Os resultados estão coincidentes com os reportados por Warchalewski et al. (2011), que indicam diminuição da quantidade de glúten presente em trigos tratados por micro-ondas e também ressalta a alta sensibilidade destas proteínas a qualquer tipo de tratamento físico ou químico sofrido.

Com exceção da temperatura de pasta que não apresentou diferença significativa $(p \leq 0,05)$ entre todas as farinhas de trigo obtidas a partir dos trigos tratadas por micro-ondas e sem tratamento, os demais parâmetros apresentaram-se diferentes significativamente, ao nível de $5 \%$ de significância, e um comportamento progressivo do perfil de viscosidade para as farinhas de trigo obtidas dos tratamentos por micro-ondas na faixa estudada.

As farinhas de trigo FM 1, FM 2 e FM 3, apresentaram maiores viscosidades máximas (Visc. Máx.) comparadas com FC, indicando que a capacidade de absorção de água pelo amido foi modificada pelo tratamento aplicado. A FC apresentou a menor quebra entre todas as amostras analisadas, indicando maior estabilidade dos grânulos de amido quando submetidos ao aquecimento, o que reflete em menores valores de viscosidade final e menor tendência à retrogradação desta farinha. Deste modo, as farinhas obtidas dos tratamentos térmicos de maiores potências aplicadas, FM 2 (450 W/18 min) e FM 3 (750 W/10 min) produziram géis com maiores viscosidades máximas (Visc. Máx.), maiores viscosidades finais (Visc. Final) e maiores tendências à retrogradação, conforme resultados apresentados.

Conforme apresentado na Tabela 3, a distribuição do tamanho médio das partículas acumuladas à $10 \%, 50 \%$ e $90 \%$ do total de partículas, indica que a farinha FC apresentou os menores valores e diferentes significativamente $(\mathrm{p} \leq 0,05)$ quando comparados com os valores obtidos para as farinhas de trigo tratadas com micro-ondas nas diferentes potências estudadas. $\mathrm{O}$ diâmetro acumulado à 90\% para as farinhas FM 1, FM 2 e FM 3, obtidas a partir dos três tratamentos por micro-ondas foram maiores que o obtido pela farinha FC (controle), sugerindo a formação de aglomerados de tamanhos maiores em decorrência da aplicação da energia de micro-ondas. Este fato é corroborado pelos maiores diâmetros médios das partículas $\left(\mathrm{d}_{4,3}\right)$ das farinhas de trigo obtidas dos trigos tratados por micro-ondas, que também apresentaram maiores valores e diferentes significativamente, ao nível de 5 \% de significância, do diâmetro médio das partículas da farinha de trigo obtida a partir do trigo controle. Apesar de não ter ocorrido diferença significativa no índice de polidispersibilidade das partículas (Span), que variou de 1,81 a 1,94, estes valores indicam (Span > 1), que as amostras são polidispersas, ou seja, há uma grande variação dos tamanhos das partículas presentes nas farinhas de trigo analisadas. Alvim et al. (2016) apresentam valores do índice de polidispersibilidade para as micropartículas de ácido ascórbico produzidas por dois métodos diferentes de 1,44 e 1,44 e concluem que estas micropartículas 
também possuem alta polidispersibilidade.

Tabela 3 - Caracterização das farinhas de trigo quanto a amido danificado, análise de glúten, propriedades de pasta e sua distribuição de tamanho de partícula e seu tamanho médio antes e após processamento por micro-ondas.

\begin{tabular}{|c|c|c|c|c|}
\hline \multirow{2}{*}{ Análises } & \multicolumn{4}{|c|}{$\operatorname{Amostras}^{1}$} \\
\hline & FC & FM 1 & FM 2 & FM 3 \\
\hline \multicolumn{5}{|c|}{ Amido danificado } \\
\hline Amido Danificado (\%) & $6,24 \pm 0,10^{\mathrm{ns}}$ & $5,54 \pm 0,67^{\text {ns }}$ & $5,81 \pm 0,03^{\mathrm{ns}}$ & $5,74 \pm 0,37^{\mathrm{ns}}$ \\
\hline \multicolumn{5}{|c|}{ Análise de glúten } \\
\hline Glúten úmido (\%) & $28,8 \pm 0,3^{\mathrm{a}}$ & $29,0 \pm 1,6^{\mathrm{a}}$ & $25,4 \pm 1,7^{b}$ & $23,6 \pm 0,7^{\mathrm{c}}$ \\
\hline Glúten seco (\%) & $9,8 \pm 0,1^{\mathrm{b}}$ & $10,31 \pm 0,1^{\mathrm{a}}$ & $8,6 \pm 0,2^{\mathrm{c}}$ & $8,0 \pm 0,2^{\mathrm{d}}$ \\
\hline Glúten index & $97,6 \pm 0,3^{\mathrm{ns}}$ & $99,2 \pm 0,9^{\mathrm{ns}}$ & $97,7 \pm 1,55^{\mathrm{ns}}$ & $96,8 \pm 3,2^{\mathrm{ns}}$ \\
\hline \multicolumn{5}{|c|}{ Propriedades de pasta } \\
\hline Temp. pasta $\left({ }^{\circ} \mathbf{C}\right)$ & $84,95 \pm 0,444^{\mathrm{ns}}$ & $84,73 \pm 0,03$ ns & $84,67 \pm 0,03$ ns & $84,72 \pm 0,08$ ns \\
\hline Visc. Máx. (cP) & $1801,3 \pm 31,8^{c}$ & $2088,0 \pm 51,6^{\mathrm{b}}$ & $2303,0 \pm 7,5^{\mathrm{a}}$ & $2334,3 \pm 15,6^{\mathrm{a}}$ \\
\hline Visc. Mín. (cP) & $1139,7 \pm 21,5^{\mathrm{d}}$ & $1250,3 \pm 15,3^{\mathrm{c}}$ & $1464,0 \pm 19,7^{b}$ & $1498,3 \pm 4,5^{\mathrm{a}}$ \\
\hline Quebra (cP) & $661,7 \pm 12,0^{\mathrm{b}}$ & $837,7 \pm 36,9^{a}$ & $829,0 \pm 14,5^{\mathrm{a}}$ & $836,0 \pm 13,7^{\mathrm{a}}$ \\
\hline Visc. Final (cP) & $2165,7 \pm 29,5^{\mathrm{d}}$ & $2385,3 \pm 54,6^{c}$ & $2676,7 \pm 7,6^{\mathrm{b}}$ & $2781,0 \pm 22,9^{\mathrm{a}}$ \\
\hline \multicolumn{5}{|c|}{ Distribuição, tamanho médio e polidispersibilidade de partícula } \\
\hline D10\% $(\mu \mathrm{m})$ & $33,21 \pm 0,76^{\mathrm{b}}$ & $38,68 \pm 1,70^{\mathrm{a}}$ & $34,61 \pm 0,83^{b}$ & $36,93 \pm 0,49^{b}$ \\
\hline D50\% $(\mu \mathrm{m})$ & $150,39 \pm 1,29^{b}$ & $157,48 \pm 2,72^{\mathrm{a}}$ & $149,79 \pm 1,73^{b}$ & $157,22 \pm 1,89^{\mathrm{a}}$ \\
\hline D90\% $(\mu \mathrm{m})$ & $305,78 \pm 5,82^{b}$ & $322,80 \pm 6,33^{\mathrm{a}}$ & $325,58 \pm 4,57^{\mathrm{a}}$ & $328,21 \pm 8,60^{\mathrm{a}}$ \\
\hline $\mathrm{d}_{4,3}(\boldsymbol{\mu m})$ & $163,76 \pm 1,68^{c}$ & $172,63 \pm 1,24^{\mathrm{a}}$ & $168,44 \pm 1,54^{b}$ & $173,50 \pm 2,37^{\mathrm{a}}$ \\
\hline Span & $1,81 \pm 0,04^{\mathrm{ns}}$ & $1,81 \pm 0,07^{\mathrm{ns}}$ & $1,94 \pm 0,03^{\mathrm{ns}}$ & $1,86 \pm 0,07^{\mathrm{ns}}$ \\
\hline
\end{tabular}

${ }^{1} \mathrm{FC}$ - farinha de trigo controle; FM 1: farinha obtida de trigo tratado com potência de $100 \mathrm{~W}$; FM 2: farinha obtida de trigo tratado com potência de $450 \mathrm{~W}$; FM 3: farinha obtida de trigo tratado com potência de $750 \mathrm{~W}$. Resultados apresentados como média \pm desvio padrão; Médias seguidas pela mesma letra na mesma linha não diferem significativamente $(\mathrm{p} \leq 0,05)$ pelo teste de Scott-Knott; ns: sem diferença significativa pela análise de variância (ANOVA). Fonte: Autores.

\section{Conclusão}

O estudo mostrou a possibilidade de utilização da tecnologia de micro-ondas para a secagem de trigo, gerada a partir de energia elétrica renovável, em substituição às técnicas de secagem convencionais, alimentadas a partir de combustíveis fósseis ou de difícil renovação, onde o sistema utilizado permitiu realizar controles de processos a fim de minimizar danos físicos ao material, como perda de rendimento de moagem, queima do grão pelo calor, controle no impacto de propriedades físico-químicas, reológicas e tecnológicas, além permitir diminuir a utilização de agentes químicos e de não gerar odores indesejáveis e compostos tóxicos aos grãos, que poderão estar presentes ao longo do processamento e transformação do trigo em farinha e demais produtos obtidos a partir desta farinha.

\section{Agradecimentos}

Dedicamos este trabalho ao Prof. Dr. Antonio Marsaioli Junior (In memorian) pelo seu incansável trabalho para o desenvolvimento da tecnologia de alimentos no Brasil. Os autores são gratos à CAPES, Coordenação de Aperfeiçoamento de 
Pessoal de Nível Superior - Brasil (CAPES) - código de financiamento 001, ao Instituto de Tecnologia de Alimentos, ao Moinho Arapongas S.A. pela doação do trigo utilizado no presente trabalho, a Fundação de Amparo à Pesquisa do Estado de São Paulo (FAPESP) pelo apoio financeiro (Processo 2014/14435-1) e ao Conselho Nacional de Desenvolvimento Científico e Tecnológico (CNPq) pela bolsa concedidas (150826/2019-2, 150826/2019-2 e 312786/2020-4).

\section{Referências}

AACCI. (2010). Approved Methods of Analysis. AACC International.

Alvim, I. D., Stein, M. A., Koury, I. P., Dantas, F. B. H., \& Cruz, C. L. de C. V. (2016). Comparison between the spray drying and spray chilling microparticles contain ascorbic acid in a baked product application. LWT - Food Science and Technology, 65, 689-694. https://doi.org/10.1016/j.lwt.2015.08.049

Baloch, U. K. (2001). WHEAT: Post-harvest Operations (D. Mejia \& B. Lewis (eds.)). FOOD AND AGRICULTURE ORGANIZATION OF THE UNITED NATIONS.

Bansal, N., Dhaliwal, A. S., \& Mann, K. S. (2015). Dielectric properties of corn flour from 0.2 to $10 \mathrm{GHz}$. Journal of Food Engineering, 166, $255-262$. https://doi.org/10.1016/j.jfoodeng.2015.06.019

Berteli, M. N. (2005). Estudo Comparativo de Processos de Secagem de Sólidos Granulados com e Sem Assistência de Microondas. Universidade Estadual de Campinas - UNICAMP.

Bressiani, J., Oro, T., Da Silva, P. M. L., Montenegro, F. M., Bertolin, T. E., Gutkoski, L. C., \& Gularte, M. A. (2019). Influence of milling whole wheat grains and particle size on thermo-mechanical properties of flour using Mixolab. Czech Journal of Food Sciences, 37(4), 276-284. https://doi.org/10.17221/239/2018CJFS

Cauvain, S. (2015). Technology of breadmaking. In Technology of Breadmaking. Springer International Publishing. https://doi.org/10.1007/978-3-319-14687-4

Chang, Y. K., Steel, C. J., \& Clerici, M. T. P. S. (2011). Use of Microwave Radiation to Process Cereal-Based Products. In S. Grundas (Ed.), Advances in Induction and Microwave Heating of Mineral and Organic Materials (First Ed., pp. 531-552). In Tech. http://www.intechopen.com/books/advances-ininduction-and-microwave-heating-of-mineral- and-organic-materials/the-use-of-microwave-radiation-energy-to-process-cereal-root-and-tuber-based- products

Clerici, M. T. P. S., Flávio Ma, Marsaioli Junior, A., \& Bertelli, M. N. (2019). Equipamento Processador Por Micro Ondas Para Tratamento De Materiais Alimentícios (Patent No. BR 102019027383 6).

Datta, A. K., \& Almeida, M. (2014). Properties Relevant to Infrared Heating of Food. In Engineering Properties of Foods (Fourth Edi, Issue 7, pp. 281-310). CRC Press, Taylor \& Francis Group.

Ferreira, D. F. (2011). Sisvar: Um sistema computacional de análise estatística. In Ciencia e Agrotecnologia (Vol. 35, Issue 6, pp. 1039-1042). https://doi.org/10.1590/S1413-70542011000600001

Hagstrum, D. W., Phillips, T. W., \& Cuperus, G. (2012). Stored Product Protection (D. W. Hagstrum, T. W. Phillips, \& G. Cuperus (eds.)). Kansas State University Agricultural Experiment Station and Cooperative Extension Service.

International Grains Council. (2018). Market Report (Issue GMR 486).

Khamis, M., Subramanyam, B., Dogan, H., \& Gwirtz, J. A. (2011). Flameless catalytic infrared radiation used for grain disinfestation does not affect hard red winter wheat quality. Journal of Stored Products Research, 47(3), 204-209. https://doi.org/10.1016/j.jspr.2010.11.005

Kumar, D., \& Kalita, P. (2017). Reducing Postharvest Losses during Storage of Grain Crops to Strengthen Food Security in Developing Countries. Foods, 6(1), 8. https://doi.org/10.3390/foods6010008

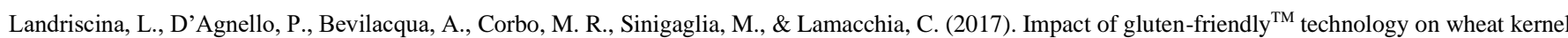
endosperm and gluten protein structure in seeds by light and electron microscopy. Food Chemistry, 221, 1258-1268. https://doi.org/10.1016/j.foodchem.2016.11.031

Li, M., Sun, Q. J., \& Zhu, K. X. (2017). Delineating the quality and component changes of whole-wheat flour and storage stability of fresh noodles induced by microwave treatment. LWT - Food Science and Technology, 84, 378-384. https://doi.org/10.1016/j.1wt.2017.06.001

Liu, C., \& Ma, X. (2016). Study on the mechanism of microwave modified wheat protein fiber to improve its mechanical properties. Journal of Cereal Science, 70,99-107. https://doi.org/10.1016/j.jcs.2016.05.018

Nirmal, R. C., Furtado, A., Rangan, P., \& Henry, R. J. (2017). Fasciclin-like arabinogalactan protein gene expression is associated with yield of flour in the milling of wheat. Scientific Reports, 7(1), 1-11. https://doi.org/10.1038/s41598-017-12845-y

Patil, S. S., Kar, A., \& Mohapatra, D. (2016). Stabilization of rice bran using microwave: Process optimization and storage studies. Food and Bioproducts Processing, 99, 204-211. https://doi.org/10.1016/j.fbp.2016.05.002

Payne, T. S. (2002). Harvest and Storage Management of Wheat. Bread Wheat: Improvement and Production. In H. Curtis, B. C.; Rajaram, S.; Gómez Macpherson (Ed.), FAO Plt. Prod. Protect. Series 30. Curtis BC, Rajaram S. Macpherson HG (eds) (p. 544). Food and Agriculture Organization of the United Nations (FAO).

Vadivambal, R., Jayas, D. S., \& White, N. D. G. (2007). Wheat disinfestation using microwave energy. Journal of Stored Products Research, 43(4), 508-514. 
Research, Society and Development, v. 10, n. 8, e12610817033, 2021

(CC BY 4.0) | ISSN 2525-3409 | DOI: http://dx.doi.org/10.33448/rsd-v10i8.17033

https://doi.org/10.1016/j.jspr.2007.01.007

Wang, T. H., \& Lu, S. (2013). Production of xylooligosaccharide from wheat bran by microwave assisted enzymatic hydrolysis. Food Chemistry, 138(2-3), 1531-1535. https://doi.org/10.1016/j.foodchem.2012.09.124

Warchalewski, J. R., Gralik, J., Grundas, S., Pruska-Kędzior, A., \& Kędzior, Z. (2011). Changes in Microwave-Treated Wheat Grain Properties. In Advances in Induction and Microwave Heating of Mineral and Organic Materials. InTech. https://doi.org/10.5772/13323

Xiqiang, Z., Wenlong, W., Hongzhen, L., Yanpeng, M., Chunyuan, M., \& Zhanlong, S. (2014). Temperature rise and weight loss characteristics of wheat straw under microwave heating. Journal of Analytical and Applied Pyrolysis, 107, 59-66. https://doi.org/10.1016/j.jaap.2014.02.003

Zhang, Z., Wang, J., Zhang, X., Shi, Q., Xin, L., Fu, H., \& Wang, Y. (2018). Effects of radio frequency assisted blanching on polyphenol oxidase, weight loss, texture, color and microstructure of potato. Food Chemistry, 248 (2017), 173-182. https://doi.org/10.1016/j.foodchem.2017.12.065 